\title{
Low Voltage Electron Holography - High Voltage Electron Holography
}

\author{
B.G.Frost, A.Thesen and D.C.Joy
}

University of Tennessee, M407 Walters Life Sci, Knoxville, TN 37996-0840

The typical set-up for low voltage holography introduced by Morton and Ramberg [1] in 1939 consists of only an electron emitter, a sample and an electron detector placed in a vacuum chamber (Fig.1). They described the most important features of the microscope, which they called point projector microscope (ppm), as follows: "As this type of microscope involves no electron- optical lens elements, the images obtained are free from the ordinary aberrations. The limit of resolution depends solely on the distribution of initial velocities of the field electrons and on Fresnel diffraction by the object, making it possible to proceed beyond the resolution of the light microscope by some orders of magnitude." At that time, however, the performance of the available electron sources was very limited. After the fabrication of a highly coherent nanotip field-emitter with great brightness [2] which operates at a very low extraction voltage (100V or less) the ppm was subject to new research [3,4]. An out of focus image of a holey carbon film acquired at 350 Volts is displayed in Fig.2. We estimate from that image the lateral coherence of our field emitter and the diameter of the tip apex using the contrast of both Fresnel fringes and Youngs fringes. In order to apply the ppm to bulk materials we design a special reflection mode of operation. Our ray tracing simulations show that we have to replace the emitter by an electron gun.

We perform high voltage electron holography using a Hitachi HF-2000 FEG TEM operated at $200 \mathrm{kV}$. The electron biprism can be placed either between the objective lens and the first intermediate lens or between the first and second intermediate lenses. For both positions a medium magnification range $(10 \mathrm{kX}-100 \mathrm{kV})$ can be achieved by only weakly exciting the first intermediate lens such that it acts as a magnifier. A ray diagram with the biprism between the objective and first intermediate lens is shown in Fig.3. Here the sample is imaged by the objective lens to the first image plane. In this step the objective lens is normally excited which preserves its good imaging qualities. Then the image is further magnified by the first intermediate lens which, in combination with the negatively biased biprism, forms the hologram. We routinely obtain 100 fringes or more with a fringe contrast of $10 \%$ or better and a field of view of about $350 \mathrm{~nm}$. These numbers are obtained from the hologram of a CMOS device (Fig.4).

\section{References}

[1] G.A. Morton and E.G.Ramberg, Phys.Rev. 56 (1939) 705.

[2] H.W. Fink, IBM J. Res. Develop. 30 (1986) 460.

[3] J.C.Spence et al. in Electron Holography, A.Tonomura et al. (Eds.), Elsevier, p267-276.

[4] This research was supported by SRC under contract number LJ413.003 and was carried out as part of the user program sponsored by the U.S. DOE under contract DE-AC05-00OR22725 with the ORNL, managed by UT-Battelle, LLC. 


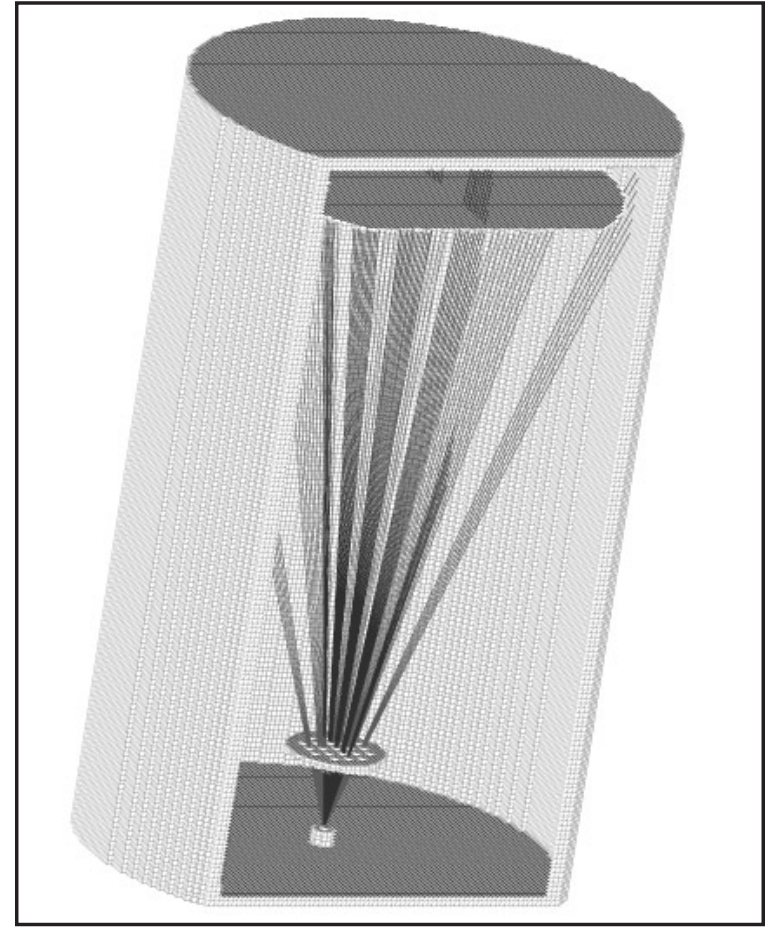

FIG.1. Ray diagram of a low voltage point projection microscope.

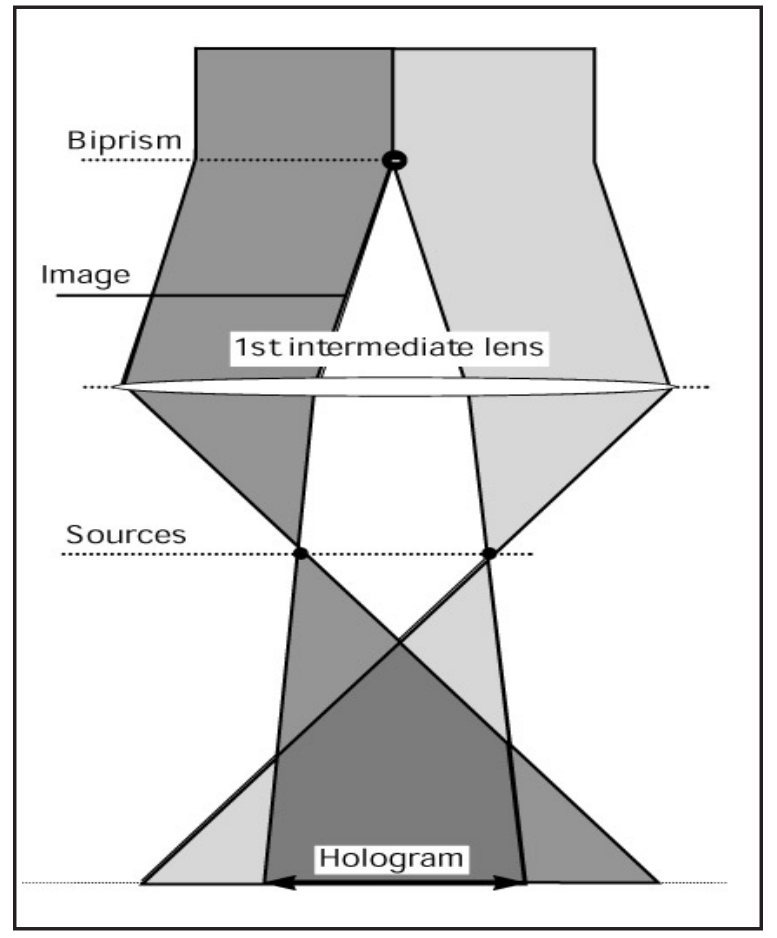

FIG.3. Ray diagram of high voltage TEM for medium magnification holography.

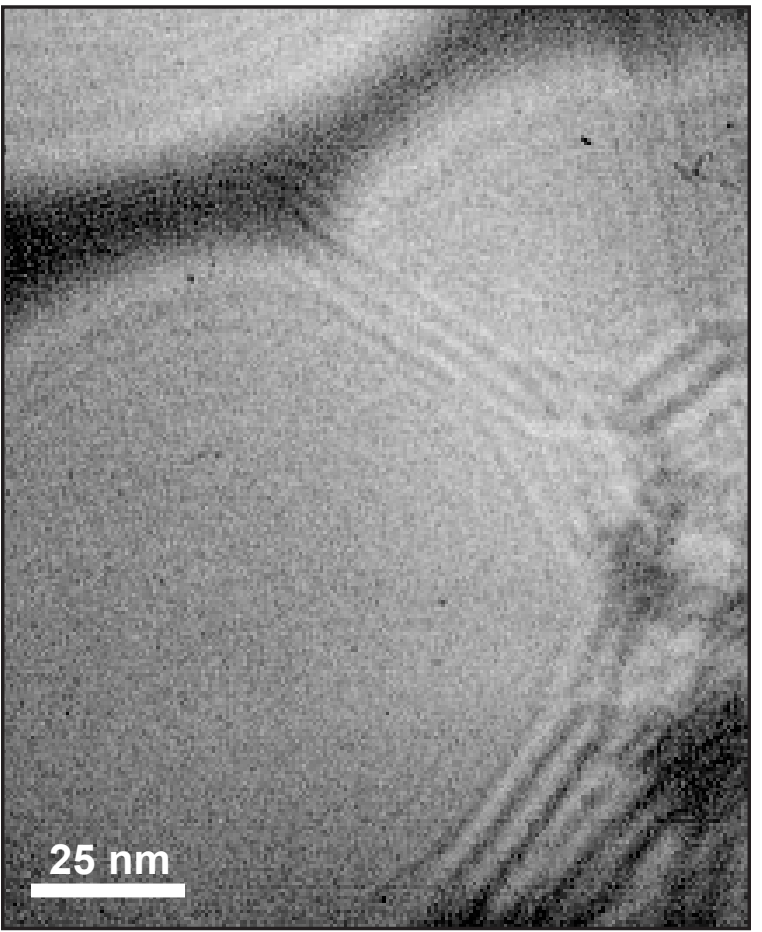

FIG.2. In-line hologram with Fresnel and Youngs fringes.

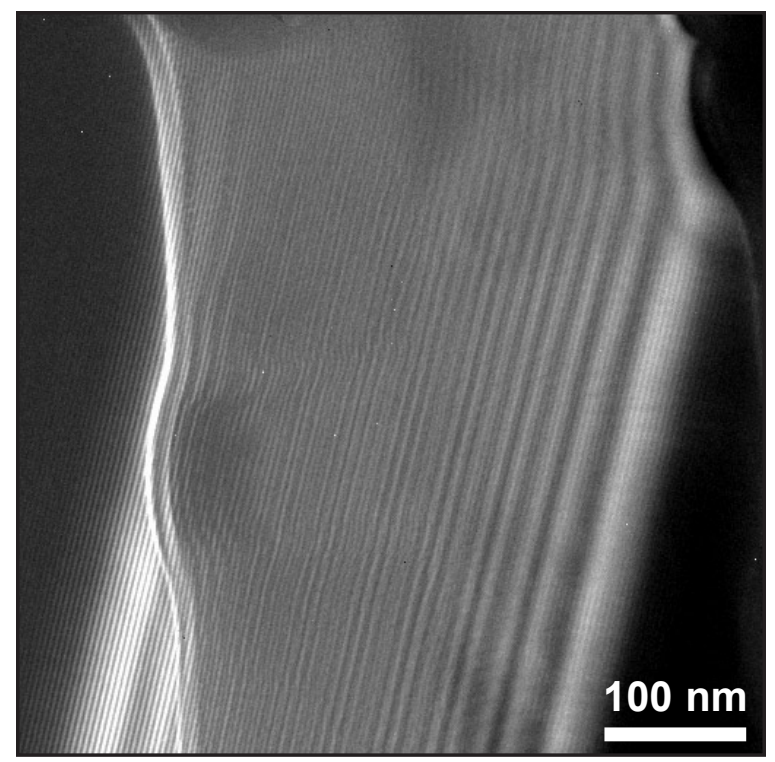

FIG.4. Hologram of transistor acquired adapting diagram of Fig.3. 